\title{
NOTE ON THE OCCURRENCE OF DIRECT FACTORS IN GROUPS
}

\author{
T. J. HEAD
}

J. Wiegold demonstrated [1] that the groups for which each normal subgroup is a direct factor are precisely the direct products (in the restricted sense) of simple groups. In this note we study the groups for which each proper normal subgroup is contained in a proper direct factor and also the groups for which each nonunit normal subgroup contains a nonunit direct factor. The result of Wiegold stated above may be regarded as a corollary to our Theorem 1. Our terms and notation are from [2].

THEOREM 1. Each proper normal subgroup of group $G$ is contained in a proper direct factor of $G$ if and only if $G$ is a direct product (restricted) of simple groups.

PRoof. Suppose that each proper normal subgroup of $G$ is contained in a proper direct factor of $G$. Let $\left[S_{\alpha}\right]_{\alpha \in A}$ be the set of all simple normal subgroups of $G$ and let $K$ be the subgroup generated by this set. We will show that $G=K$. Suppose $h \in G \backslash K$. Let $M$ be a normal subgroup of $G$ maximal subject to: $h \notin M \supset K$. Let $M^{\prime}$ be a proper direct factor of $G$ containing $M$. Let $G=M^{\prime} \times S$. If $h$ were in $M^{\prime}$ we would have the contradiction of the maximality of $M: h \notin M \times S$. Thus $h \notin M^{\prime}$ and by the maximality of $M, M^{\prime}=M$. Let $N$ be a nonunit normal subgroup of $S$. Since the only direct factors containing $M$ are $M$ and $G$ we have $M \times N=G$ and consequently $N=S$. We have the contradiction: $S$ is a simple normal subgroup of $G$ which is not contained in $K$. We conclude that $G=K$. By Zorn's lemma there is a subset $B$ of $A$ for which $G=\prod_{\alpha \in B} S_{\alpha}$.

Conversely, if $G=\prod_{\alpha \in B} S_{\alpha}$ where each $S_{\alpha}$ is simple and if $N$ is a normal subgroup of $G$ then the family of those subsets $C \subset B$ for which the product $N \times \prod_{\alpha \in C} S_{\alpha}$ is direct is closed under the operation of forming unions of inclusion chains. By Zorn's lemma there is a subset $D \subset B$ which is maximal subject to the requirement that the product $N \times \prod_{\alpha \in D} S_{\alpha}$ be direct. By the maximality of $D$ and the simplicity of each $S_{\alpha}$ we have $S_{\alpha} \cap N \times \prod_{\alpha \in D} S_{\alpha}=S_{\alpha}$ for every $\alpha \in B$. Consequently $G=N \times \prod_{\alpha \in D} S_{\alpha}$ which shows that $N$ is a direct factor of $G$.

TheOREM 2. A group $G$ is isomorphic to a subdirect product of a

Received by the editors January 3, 1963. 
family of simple groups if and only if for each nonunit normal subgroup $N$ of $G$ there is a proper normal subgroup $N^{\prime}$ of $G$ for which $N N^{\prime}=G$.

Proof. Suppose $G$ is a subdirect product of simple groups. Suppose $N$ is a nonunit normal subgroup of $G$. Let $1 \neq x \in N$ and let $N^{\prime}$ be a maximal normal subgroup of $G$ not containing $x$. The existence of such a maximal normal subgroup follows from the fact that each of the simple groups under discussion is a homomorphic image of $G$. We have $N N^{\prime}=G$.

Conversely, let $G$ be a group for which for each nonunit normal subgroup $N$ of $G$ there is a proper normal subgroup $N^{\prime}$ of $G$ for which $N N^{\prime}=G$. Suppose $1 \neq x \in G$. Let $N$ be the normal subgroup of $G$ generated by $x$ and let $N^{\prime}$ be a proper normal subgroup of $G$ for which $N N^{\prime}=G$. Let $M$ be a normal subgroup of $G$ maximal subject to: $x \notin M \supset N^{\prime}$. Such an $M$ must be a maximal normal subgroup of $G$. Thus each nonunit element of $G$ lies outside a maximal normal subgroup of $G$, and $G$ is therefore isomorphic to a subdirect product of a family of simple groups.

CoROLlaRy. If each nonunit normal subgroup of a group $G$ contains a nonunit direct factor of $G$ then $G$ is isomorphic to a subdirect product of a family of simple groups.

Proof. For each nonunit normal subgroup $N$ of $G$ we have a direct decomposition $G=H \times N^{\prime}$ where $H \subset N$ and $N^{\prime} \neq G$. Then $N N^{\prime}=G$. By Theorem 2, $G$ is isomorphic to a subdirect product of simple groups.

Our final theorem has discouraged us from trying to determine the structure of those groups $G$ having the property that each nonunit normal subgroup contains a nonunit direct factor. The proof is based on the following theorem which appears, for instance, as Theorem 16 in [3]: Any group can be embedded in a noncyclic simple group.

Theorem 3. Any group $A$ can be embedded as a semidirect factor in a group $G$ having the property that each nonunit normal subgroup of $G$ contains a nonunit direct factor of $G$.

Proof. Let $A$ be a group. Embed $A$ in a noncyclic simple group $S$. Let $P=\prod^{*} S_{i}$ (unrestricted direct product, $i$ a natural number) where $S_{i}=S$ for each $i$. Let $W=\prod S_{i}$ (restricted direct product) and regard $W$ as a subset of $P$ in the natural way. For each $b \in A$ let $\bar{b}$ be that element of $P$ which has $b$ as its $i$ th component for each $i$. The elements $\{b \mid b \in A\}$ form a subgroup $\bar{A}$ of $P$ which is isomorphic to $A$. 
Identify $\bar{A}$ with $A$. Let $G$ be the subgroup of $P$ generated by $W$ and $A$. $W$ is normal in $G$ and $W \cap A=1$. Thus $G=W A$ is a semidirect product of $W$ and $A$. Let $N$ be any nonunit normal subgroup of $G$. Let $1 \neq x \in N$. Let $i$ be such that the $i$ th component, $x_{i}$, of $x$ is not 1 . Since $S_{i}$ is a centerless group there is a $y_{i} \in S_{i}$ for which $x_{i} y_{i} \neq y_{i} x_{i}$. Let $y$ be the element of $P$ which has $i$ th component $y_{i}$ and $j$ th component 1 for $j \neq i$. $y \in W \subset G$. Then $1 \neq x^{-1} y^{-1} x y \in N \cap S_{i}$. Since $S_{i}$ is simple $N \supset S_{i}$ which is a direct factor of $G$.

\section{BIBLIOGRAPHY}

1. J. Wiegold, On direct factors in groups, J. London Math. Soc. 35 (1960), 310-320.

2. A. G. Kurosh, The theory of groups. I, Chelsea, New York, 1955.

3. W. R. Scott, The infinite symmetric and alternating groups, Univ. Kansas Rept. 5 (1956), 1-22.

Iowa State University 